\title{
ATENÇÃO À SAÚDE: CONCEPÇÕES DE PROFISSIONAIS DO CENTRO DE ATENÇÃO PSICOSSOCIAL ÁLCOOL E DROGAS
}

EALTH CARE: DESIGN OF PROFESSIONALS FROM THE PSYCHOSOCIAL CARE CENTER AND DRUGS

\section{Joze Karlem da Silva Teixeira', Marlene Gomes Terra², Daiana Foggiato de Siqueira ${ }^{3}$, Keity Laís Siepmann Soccol ${ }^{4}$, Jael Maria de Aquino ${ }^{5}$, Valquíria Toledo Souto ${ }^{6}$}

RECEBIDO EM: 25/07/2019 | ACEITO EM: 10/01/2020

DOI: $10.5902 / 2317175839234$

\section{RESUMO}

Objetivo: Apreender as concepções de profissionais de saúde do Centro de Atenção Psicossocial Álcool e Outras Drogas sobre a atenção à saúde. Método: estudo qualitativo, no qual participaram 15 profissionais de saúde de dois Centros de Atenção Psicossocial Álcool e Outras Drogas de um município do interior do estado do Rio Grande do Sul, Brasil. Para análise utilizou-se a Proposta Operativa de Minayo. Resultados: A partir da análise das informações emergiram duas categorias: atenção à saúde dos usuários de álcool e outras drogas e percepção dos profissionais sobre as políticas públicas de saúde. Conclusões: torna-se importante elucidar as concepções dos profissionais de saúde dos Centros de Atenção Psicossocial Álcool e Outras Drogas, visando realizar o planejamento e desenvolvimento de estratégias e implementação de mudanças para as ações de saúde mental que sejam voltadas as necessidades dos(as) usuários(as) de drogas.

Palavras-chave: Atenção a Saúde. Usuários de Drogas. Transtornos Relacionados ao Uso de Substâncias. Profissionais de Saúde.

\footnotetext{
1 Enfermeira. Mestre em Enfermagem pela Universidade Federal de Santa Maria. Especialista em Saúde Mental pelo programa de Residencia Integrada em Saúde Mental da Universidade do Vale do Rio dos Sinos. Lattes: http://lattes.cnpq.br/4348400193412751

Universidade do Vale do Rio dos Sinos. Brasil

2 Enfermeira. Doutora em Enfermagem. Professora do programa de pós-graduação de Enfermagem da Universidade Federal de Santa Maria. Lattes: http://lattes.cnpq.br/0373218283700156

Universidade Federal de Santa Maria. Brasil

3 Enfermeira. Doutora em Enfermagem. Professora Adjunta do Departamento de Enfermagem da Universidade Federal de Santa Maria. ORCID iD: https://orcid.org/0000-0002-8592-379X.

Lattes: http://lattes.cnpq.br/7655153358143882

Universidade Federal de Santa Maria. Brasil

4 Enfermeira. Doutora em Enfermagem. Professora da Universidade Franciscana.

Lattes: http://lattes.cnpq.br/8288461290333674

Universidade Franciscana. Brasil

5 Enfermeira. Doutora em Enfermagem Psiquiátrica e Pós-doutorado em enfermagem pela Universidade Federal de Santa Maria. Professora adjunto da Faculdade de Enfermagem Nossa Senhora das Graças da Universidade de Pernambuco, integra o corpo docente do Programa Associado de Pós - Graduação em Enfermagem. ORCID iD: http://orcid.org/0000-0002-6949-7217

Lattes: http://lattes.cnpq.br/1986461838794888

Faculdade de Enfermagem Nossa Senhora das Graças da Universidade de Pernambuco. Brasil

6 Enfermeira. Docente do Curso de Graduação em Enfermagem da Faculdade Dom Alberto. Doutoranda do Programa de Pós-Graduação em Enfermagem/Universidade Federal de Santa Maria.

Lattes: http://lattes.cnpq.br/0987544849474283

Instituição/ Afiliação: Faculdade Dom Alberto. Brasil
} 


\section{ABSTRACT}

Objective: To understand the conceptions of health professionals of the Psychosocial Care Center Alcohol and Other Drugs about health care. Method: qualitative study, in which 15 health professionals from two Psychosocial Care Centers Alcohol and Other Drugs from a municipality in the interior of the state of Rio Grande do Sul, Brazil, participated. For analysis we used the Minayo Operative Proposal. Results: From the analysis of the information, two categories emerged: attention to the health of users of alcohol and other drugs and professionals' perception about public health policies. Conclusions: It is important to clarify the conceptions of health professionals of the Psychosocial Care Centers Alcohol and Other Drugs, aiming to carry out the planning and development of strategies and implementation of changes for mental health actions that are geared to the needs of drug users.

Keywords: Health Care. Drug users. Substance-Related Disorders. Health professionals.

\section{Introdução}

No Brasil, as políticas sobre álcool e outras drogas tiveram, inicialmente, um caráter de repressão em relação ao uso e ao usuário. Essa ideia está pautada na concepção da doença e do proibicionismo, o que promove um modelo de atenção pautado em estigmas e rótulos por parte da sociedade, dificultando o acesso dos usuários de drogas, bem como de seus familiares aos serviços de saúde. Isto reafirma que essa política trabalha na perspectiva da redução da oferta (RO) e está focada na lógica da abstinência (abstenção ao uso de drogas), ou seja, busca alcançar o ideal de uma sociedade protegida do uso de drogas ilícitas e do uso indevido de drogas lícitas (BRASIL, 2005).

Entretanto, outra política orienta para a atenção integral a saúde aos usuários de álcool e outras drogas que direciona para um modelo de atenção enfocada na singularidade dos usuários, em que a abstinência não é o único objetivo a ser alcançado. E, quando se trata de prestar atenção à saúde das pessoas, os profissionais necessitam aprender a respeitar as escolhas que os usuários realizam. As práticas de atenção à saúde precisam levar em conta às diversidades, acolhendo sem julgamentos a cada situação e, estimulando sua participação no cuidado (BRASIL, 2004), incluindo considerar à perspectiva da Redução de Danos (RD).

Em relação às políticas de atenção ao usuário de álcool e outras drogas, nos serviços de saúde mental, pode ser identificado os dois modelos de atenção, em que alguns profissionais adotam a RO, ainda focados na abstinência e, outros aderem à $\mathrm{RD}$ realizando uma sistematização das necessidades a serem atendidas, associando ao meio cultural e à comunidade a qual os usuários estão inseridos. Estudos realizados com enfermeiros evidenciam que os mesmos percebem os usuários de drogas como delinquentes, doentes, manipuladores, sem limites e responsáveis por sua doença. Entretanto, percebeu-se também que os usuários são vítimas das desigualdades sociais (SPRICIGO; ALENCASTRE, 2004; VARGAS et al., 2013).

Diante desse contexto, torna-se relevante realizar estudos que busquem ampliar essa discussão, considerando que é uma prática de serviços públicos 
que devem estar em constante monitoramento e avaliação para qualificação dos próprios serviços e profissionais da saúde. Assim, tem-se como questão norteadora: Quais as concepções de profissionais de saúde de Centro de Atenção Psicossocial Álcool e Outras Drogas acerca da atenção à saúde? E, como objetivo: apreender as concepções de profissionais de saúde de Centro de Atenção Psicossocial Álcool e Outras Drogas acerca da atenção à saúde.

\section{Metodologia}

Estudo qualitativo que considera a singularidade do sujeito e é capaz de incorporar a questão do significado e da intencionalidade, como sendo essenciais as relações, as ações e estruturas sociais respeitando as suas especificidades culturais (MINAYO, 2014). Os cenários foram dois CAPS ad de um município do estado do Rio Grande do Sul, Brasil. Estes locais foram selecionados por serem serviços de saúde que prestam assistência a pessoas que apresentam problemas decorrentes do uso de drogas com idades acima de 12 anos. $O$ atendimento nos CAPS ad é realizado por territorialidade, ou seja, cada serviço atende diferentes territórios (bairros) do município.

Participaram 15 profissionais de saúde dos CAPS ad (dois assistentes sociais, dois técnicos de enfermagem, um fisioterapeuta, dois técnicos em saúde mental, quatro psicólogos, dois médicos e dois enfermeiros), mulheres e homens, com idades entre 24 e 61 anos. Após a concordância em participar do estudo, eles assinaram o Termo de Consentimento Livre e Esclarecido (TCLE) em duas vias, sendo que uma delas ficou em sua posse. Para garantir o anonimato, utilizou-se a letra "P" acompanhada de um número (P1, P2, P3..., P15).

Elegeu-se como critérios de inclusão: ser profissional da saúde, servidor municipal ou Residente Multiprofissional em Saúde, que estivessem atuando nos CAPS ad durante o período da coleta de dados. E, os de exclusão: os profissionais de saúde que estivessem em licença para tratamento à saúde e licença à maternidade no período da coleta de dados.

Utilizou-se as entrevistas semiestruturadas, em áudio gravadas e transcritas, no período de março a maio de 2015, realizadas em uma das salas dos CAPS. Para dar inicio as entrevistas, realizou-se um sorteio entre os dois CAPS ad sendo convidado o primeiro participante e, na sequência, o do outro serviço. Para iniciar as entrevistas utilizou-se as questões: Como você vê o trabalho desenvolvido pelos profissionais de saúde na atenção à saúde aos usuários de drogas no CAPS ad? O que você pensa sobre as Políticas de Atenção à Saúde de pessoas usuárias de álcool e outras drogas em tratamento no CAPS ad?

As informações foram submetidas a Análise Temática da Proposta Operativa de Minayo. Esta se caracteriza em dois momentos: o primeiro, fase exploratória (compreende o mapeamento e as determinações fundamentais da pesquisa); e, o segundo, interpretativo, que se constitui em etapas: a ordenação dos dados e a classificação dos dados, categorização e análise final (MINAYO, 2014). 
Para o desenvolvimento desta pesquisa com seres humanos, foram respeitados os princípios da Resolução N 466/2012 do Conselho Nacional de Saúde (BRASIL, 2012a). A presente pesquisa teve aprovação do Comitê de Ética sob o número 981.656 .

\section{Resultados e discussões}

Emergiram duas categorias: atenção à saúde dos usuários de álcool e outras drogas; e, percepção dos profissionais sobre as políticas públicas de saúde.

Atenção à saúde dos usuários de álcool e outras drogas

Esta categoria foi formada pelos temas: abstinência, redução de danos, questão social, intersetorialidade e reinserção social. Uma das concepções apontadas pelos profissionais acerca da atenção em saúde foi a dificuldade que o usuário tem em manter-se em abstinência e ter possíveis recaídas.

\section{A gente acha que o paciente vai conseguir manter a abstinên- cia e quando vê ele recai [...] a gente como profissional, assim quando o paciente recai fica bem, bem chateado porque tem que começar tudo de novo. (P2.)}

A alternativa de cuidado pautados na lógica da RD e da abstinência aos usuários de drogas vem perpassando por diversas discussões na atualidade sobre os melhores resultados de tais abordagens. Neste contexto, tem-se a abstinência que é a abstenção do uso de bebidas alcoólicas ou de outras drogas (SENAD, 2010). E, a recaída significa que a pessoa voltou a fazer uso de algum tipo de droga, após estar por um período de tempo em abstinência. Essa percepção da abstinência como exclusiva possibilidade de tratamento desvia o foco da atenção à saúde, que é o usuário (FORTESKI, 2013).

$\mathrm{Na}$ atenção à saúde a esses usuários, ainda após a Reforma Psiquiátrica (Lei N 10.216), há profissionais que prestam atendimento na lógica da abstinência, pois acreditam que o modo de cuidar está centrado na questão da droga, ou seja, de forma reducionista e fragmentada (ASSIS; BARREIROS; CONCEIÇÃO, 2013). Entretanto, identificam-se profissionais de saúde que reconhecem a abstinência como não sendo a única ou a mais adequada opção de tratamento.

Não quer dizer que eles deixem de usar. Alguns sim estão em abstinência, mas a maioria fica um, dois anos e tem uma recaída ou dois, três meses e tem uma recaída. Mas, na verdade é um uso que não chega a prejudicar a vida deles como prejudicava antes e, isso ai, para mim já é uma vitória. (P11.)

O participante, acima, mostra a RD como alternativa que atenda a pessoa na sua singularidade, pois trabalhar nessa perspectiva é buscar um cami- 
nho junto aos usuários traçando objetivos e escolhas mais adequadas para o seu tratamento. Esta compreensão passa pelo contexto individual, coletivo e social (CRUZ, 2011). Na RD, a abstinência poderá ser uma meta a ser alcançada, porém é pactuada com os usuários e não uma regra imposta pelos profissionais de saúde. $E$, tem como objetivo um olhar ampliado e pautado em realizar intervenções singulares (PASSOS, 2011; MAYER, 2013).

A RD é uma estratégia que auxilia nas práticas de cuidado à saúde que diminua os danos ocasionados pelo uso abusivo de drogas para os usuários e grupos sociais envolvidos (CRUZ, 2011). Também, possibilita ao profissional de saúde ampliar o olhar para além das drogas buscando atender as necessidades dos usuários. E, necessita considerar as questões sociais no contexto de vida, as familiares e as de saúde.

[...] tem todo o aspecto social, que é bem difícil, pacientes [...] desempregados, pacientes de rua, pacientes já sem família (família não apoia), onde o prognóstico, assim, é bem ruim. (P12).

O contexto social dessas pessoas pode ser considerado um problema emergente que contribui com o uso de drogas. Este poderá estar associado a sofrimentos decorrentes da falta de cidadania, acesso a bens materiais e a valores sociais. No entanto, os problemas relacionados com as drogas, às vezes, acabam sendo visto como desvios das normas sociais e não como manifestações dos problemas sociais (TORCATO, 2013).

O uso das drogas pode gerar para os usuários algumas dificuldades, por exemplo, a falta de diálogo familiar, o desemprego e a vulnerabilidade a situação de rua. Estas suscitam demandas peculiares aos profissionais da saúde para prestarem assistência a estes usuários. Para tanto, é preciso articular as políticas de saúde com as sociais, da justiça, da educação, da segurança, levando em consideração à história de vida dos usuários (PEREIRA, 2012). E, também, identifica-se uma série de articulações intersetoriais para suprir essas demandas no que tange aos serviços sociais essenciais como: assistência social, habitação, cultura, entre outros (PEREIRA, 2012; SILVA, 2014).

A intersetorialidade é entendida como uma estratégia para resolver ou minimizar problemas que ultrapassam o âmbito de atuação de um só setor e passou a ser um dos requisitos para a implementação das políticas setoriais, visando sua efetividade por meio da articulação entre as instituições e saberes. Para tanto, é necessário oferecer cuidado por meio de estratégias de acolhimento e de Clinica Ampliada compartilhados com a rede estabelecendo políticas transversais aos diferentes setores. Também, poderá trazer ganhos para a comunidade, abrindo olhares para a superação da fragmentação na atenção e articulação das políticas públicas (BRASIL, 2010; NASCIMENTO, 2010; SILVA, 2014).

Assim, tornam-se necessárias estratégias que promovam a relação entre 
os profissionais dos diferentes setores por meio da comunicação, interação e compartilhamento de conhecimentos em torno de objetivos em comuns (FERNANDES, 2013). É preciso estruturar-se para trabalhar de forma integrada e coerente com a reinserção social dos usuários (GANEV, 2011):

Eu sempre dei ênfase para a questão da reinserção social e muito pouco para as drogas. (P15).

A reinserção social compreende a auto exclusão, que acontece a partir do usuário para consigo mesmo; e, a exclusão social na qual o usuário distancia-se das condições mínimas de vida, que inclui o trabalho, educação, moradia e saúde. Assim, o processo de reinserção social começa pelo acolhimento do usuário estendendo-se ao trabalho interdisciplinar e qualificado dos profissionais dos serviços (GANEV, 2011). E, é uma das prioridades da Política do Ministério da Saúde para a atenção integral a usuários de álcool e outras drogas (BRASIL, 2004). Logo, é importante promover ações de reinserção social que tenham sentido para os usuários conforme suas necessidades.

Percepção dos profissionais sobre as políticas públicas de saúde

Esta categoria é composta pelos temas: desconhecimento dos profissionais, falta de interesse em apropriar-se acerca das políticas, dificuldade em concretizar na prática as políticas, gestão das políticas no município, assistência na lógica manicomial, desinstitucionalização e trabalhando em consonância com a Política Nacional de Humanização.

Percebe-se desconhecimento por parte de alguns profissionais acerca das Políticas Públicas relacionadas à saúde dos usuários de drogas. Além disto, observa-se a falta de interesse de alguns em atualizar-se nessa temática. Apesar deles saberem da existência das Políticas Públicas de atenção integral à saúde dos usuários de drogas, alguns reconhecem que não estão a par do que preconiza a Política:

Essas politicas existem, só que muitas vezes o que acontece é que os profissionais não estão a par dessas politicas [...] tem alguns profissionais que não tem muito conhecimento dessas políticas. (P10).

A falta de interesse de alguns dos profissionais de saúde pela atualização na sua área sinaliza para falta de resolutividade nos serviços de saúde mental. Além disto, aponta limitações para atuar na rede de saúde em consonância com a Política sobre Álcool e Outras Drogas. Isso implica em dificuldades para realizar o cuidado ao usuário de drogas e reafirma a lógica reducionista (CORTES et al., 2014).

A fala de P5, abaixo, mostra a sensibilidade do profissional ao identificar a sua fragilidade em relação ao conhecimento da Política e a sua dificuldade de associar teoria e prática. 
Eu te digo que eu sou bem leiga ainda nessas políticas. Eu me vejo com bastante dificuldade de associar teoria e prática [...]. (P5).

Os profissionais nos serviços de saúde precisam investir em si mesmo, por meio de cursos de formação permanente que os atualizem acerca da temática. Nesse sentido, tem-se a Política Nacional de Educação Permanente em Saúde (EPS), que se fundamenta na aprendizagem significativa e na possibilidade de transformação das práticas profissionais, pois acontece no cotidiano dos profissionais e a partir necessidades dos serviços (BRASIL, 2009; TOLENTINO et al., 2018). Entretanto, os profissionais relatam que as Políticas Públicas são bem elaboradas, porém apresentam dificuldade em concretizá-las na prática profissional pelo distanciamento da teoria com a realidade dos serviços.

É, eu vejo que na escrita ela é muito linda, muito perfeita, mas na hora da gente botar em prática é bem complicado [...]. Então, na Lei, a politica ela existe. Ela (a política) é bonita no papel, mas assim, quando vai se aplicar é bem difícil. (P14).

$\mathrm{Na}$ fala, acima, identifica-se que a política é adequadamente formulada e preconiza as necessidades da população. Porém, a questão é como realizar o cuidado aos usuários nos serviços de saúde, pois na prática, os profissionais deparam-se com dificuldades relacionadas a recursos materiais, financeiros e humanos. Para que a atenção à saúde, preconizada, efetive-se na prática, não basta apenas estarem escritos nas políticas de saúde. Depende de ações desenvolvidas pelos serviços de saúde em consonância com a comunidade e voltadas as reais necessidades da população (VAZ, 2011).

É importante lembrar que um profissional não se constitui apenas de teoria, embora seja importante. Ele vai se constituindo como profissional na relação existente entre a teoria e a prática. Com relação a isso, um estudo mostra que a indissociabilidade entre estes campos possibilita a construção de ações conjuntas entre as formas de arquitetar e organizar o trabalho em saúde (BRASIL, 2010). A seguir, P6 traz a Portaria Nº 3.088 de 2011, que visa articular as Redes de Atenção Psicossocial (RAPS) (BRASIL, 2011a).

Eu acho que no papel não falta [...] o que falta é isso ser operacionalizado [...] que deveria já estar impactando, a 3.088 que é de 2011. Falta política? Não! Falta é dispositivo e mobilizar essa rede, fazer com que ela funcione. (P6).

A RAPS tem a finalidade de criação, ampliação e articulação dos pontos de atenção à saúde para pessoas com sofrimento ou transtorno mental e com necessidades decorrentes do uso de drogas, no âmbito do SUS. Destaca-se que fazem parte dessa rede: Atenção Básica em Saúde, Atenção Psicossocial Especializada, Atenção de Urgência e Emergência, Atendimento Residencial de Caráter Transitório, Atenção Hospitalar, Estratégias de desinstitucionalização, 
Reabilitação Psicossocial. Identifica-se que todos esses serviços fazem parte da RAPS, o que teoricamente deveria dar suporte para todos os usuários, em todos os níveis de complexidades (BRASIL, 2011a).

Sendo assim, deve haver uma coerência entre a situação de saúde e o sistema de atenção à saúde prestada. $E$, quando não se tem essa coerência, dificulta prestar uma assistência de qualidade. Instala-se, assim, uma crise nos sistemas de atenção à saúde (MEDINA, 2014):

Questão da gestão, ela devia envolver todas as secretarias, porque a dependência química ela não é um problema só de saúde. Quando chega na saúde a doença já está instalada, quer dizer muitas outras áreas poderiam atuar para evitar que se chegasse, porque é muito uma questão social. (P13).

As críticas à gestão em saúde são diversas e apontam a dimensão dos problemas a serem enfrentados pelos municípios. Sabe-se que existem serviços de saúde com fragilidades na gestão o que dificulta oferecer uma assistência resolutiva e de qualidade aos usuários dos serviços. Os desafios para a gestão é a consolidação de um sistema de saúde, que responda às demandas e necessidades de saúde da população. Salienta-se a importância dos gestores reverem as formas de gestão em saúde no sentido que as práticas sejam alicerçadas na cooperação e na interdisciplinaridade em que os profissionais e os usuários operem como sujeitos ativos (LORENZETTI et al, 2014).

Para tanto, é importante desenvolver estratégias de atenção e formação em saúde em consonância com a gestão, que possam contribuir com a articulação em rede entre os serviços com a finalidade de melhorar a comunicação entre os profissionais da área de saúde mental. Além disto, busca-se fortalecer a rede de atenção à saúde mental, que possa propiciar acolhimento e um tratamento respeitando a autonomia do usuário (CORTES et al, 2014).

Percebe-se questões que influenciam na qualidade da atenção a saúde dos usuários no que tange a dinâmica de trabalho realizada por alguns profissionais. Estes, ainda, prestam uma assistência pautada na lógica manicomial contrariando ao que é preconizado na da Reforma Psiquiátrica, Lei № 10.216.

Está realmente indo muito contra o que se batalha tanto nessa Reforma Psiquiátrica. Assim, esse processo de desinstitucionalizaçãoque a gente tanto lutou, a gente, eu digo, os profissionais num momento em que isso não está acontecendo e cada vez mais está se trabalhando para se institucionalizar as pessoas, torná-las doentes e torná-las realmente dependentes de alguma patologia, um CID. (P1).

É importante lembrar que a Reforma Psiquiátrica possibilitou ter um olhar diferenciado, favorecendo o cuidado humanizado e individualizado aos usuários. Este vai além dos aspectos da saúde, pois considera todos os âmbi- 
tos que perpassam a vida dos usuários, propiciando a sua autonomia. Neste contexto, tem-se a desinstitucionalização que é uma alternativa para se desconstruir as práticas manicomiais e possibilitar um cuidado em liberdade e no território dos usuários (GUEDES, 2010; TOLENTINO et al., 2018).

Eu acho que as nossas políticas já têm algumas coisas que estão meio defasadas, meio que não foram reajustadas digamos assim, por exemplo, quanto mais fora, quanto mais desinstitucionalizado o usuário, melhor! (P9).

Com relação ao exposto, fica claro nas discussões que as Políticas Públicas relacionadas ao uso de Álcool e outras Drogas no papel são muito boas, bem elaboradas e, teoricamente, teriam que dar suporte às necessidades dos usuários. Entretanto, os profissionais alegam que ainda têm entraves a serem vencidos, tanto por parte deles quanto da sociedade.

No que tange a Política Nacional de Humanização (PNH), os profissionais de saúde percebem que a Atenção Básica $(A B)$ é a porta de entrada dos serviços de saúde e exerce um papel fundamental no atendimento aos usuários realizando o primeiro acolhimento.

A questão do acolhimento na Atenção Básica é importante. (P6).

O acolhimento compreende a recepção do usuário pelos profissionais de saúde desde a sua chegada na Unidade de Atenção Básica de Saúde até a resolutividade de suas necessidade de saúde. O profissional precisa responsabilizar-se integralmente pelo usuário ouvindo suas queixas, suas preocupações e angústias. E, quando necessário intermediar a articulação com os outros serviços de saúde para a continuidade da assistência (BRASIL, 2006). Assim, os profissionais de saúde que exercem suas ações na $A B$ têm o papel fundamental de acolher, amenizar os problemas decorrentes de saúde dos usuários.

O acolhimento é um instrumento que possibilita aos profissionais de saúde ter um olhar integral do usuário. Um bom acolhimento por parte dos profissionais de saúde aos usuários permitirá uma melhor compreensão das necessidades deles (RIBEIRO, 2010). Quanto mais os profissionais de saúde conhecem os usuários na sua integralidade, mais dinâmico e resolutivo será o atendimento. Nessa perspectiva, as ações de cuidado aos usuários de drogas precisam acontecer na lógica da integralidade incluindo toda a rede de atenção à saúde e articulando-se com outras políticas (CLEMENTE; LAVRADOR; ROMANHOLI, 2013).

Destaca-se que um dos pilares da $A B$ é o principio da integralidade, que se baseia em ações de promoção, prevenção de agravos e recuperação da saúde dos usuários. A integralidade possibilita uma visão singular do sujeito, considerando o seu contexto histórico, familiar, social e ambiental em que estão inseridos (SOUZA, 2012): 
Tem que apostar realmente na vida na comunidade [...] melhorar ampliar o cuidado da Atenção Básica, assim com ações ali, que possam até dar conta e que não precise de uma ação secundária que são os CAPS. (P1).

A $A B$ caracteriza-se por um conjunto de ações de saúde, no âmbito individual e coletivo, que abrangem a promoção e a proteção da saúde, a prevenção de agravos, o diagnóstico, o tratamento, a reabilitação e a manutenção da saúde (LAVRAS, 2011). Para tanto, os profissionais de saúde necessitam estar constantemente em busca de atualizações para suprir as demandas do serviço.

Nesse contexto, destaca-se a importância da Educação Permanente nos serviços de saúde, a fim de dar suporte a esses trabalhadores. A Educação Permanente é uma estratégia essencial para a reformulação das práticas de atenção, visto que o processo de aprendizagem tem natureza participativa e tem como eixo fundamental o cotidiano dos serviços de saúde (BRASIL, 2005; TOLENTINO et al., 2018).

Seria interessante que nós tivéssemos nas unidades de saúde, profissionais capacitados na saúde mental para dar esse apoio. $O$ paciente não precisava, por exemplo, vir ao CAPS a primeira vez, vai na Unidade Básica e tem um primeiro atendimento lá. (P2.)

O primeiro atendimento ao usuário de drogas deveria ser realizado na $A B$ e, se necessário encaminhado a um serviço de atenção secundária como os CAPS. Porém, mesmo se encaminhado a um serviço especializado, não exclui a responsabilidade da $A B$ por esse usuário, devendo haver diálogo entre essas instâncias. É necessário trabalhar em articulação com os serviços de saúde, discutindo práticas de cuidado que sejam resolutivas para assegurar a continuidade e integralidade na assistência.

Essa continuidade e integralidade na qual se refere, possibilita uma percepção abrangente da pessoa, considerando seu histórico de vida, social, familiar e ambiental em que está inserido (SOUZA, 2012; SIQUEIRA et al., 2019). Nessa perspectiva de proporcionar uma assistência integral aos usuários, justifica-se a importância da inserção dos profissionais dos serviços secundários nos serviços de atenção primária, uma vez que, os mesmos estão dando suporte especializado para a $A B$.

Tu tens que ter um olhar biopsicossocial, espiritual. [...] E, é isso que a gente esta tentando enquanto apoiadora (matriciamento) nas Estratégias de Saúde da Família, fazer com que eles tenham esse olhar um pouquinho diferenciado sobre a saúde mental do paciente. (P9).

Dessa forma, como apoiadores dos serviços primários, os profissionais com experiência em saúde mental, em especial com usuários de drogas, buscam auxiliar e abrir possibilidades de atenção para esses usuários na atenção básica. 
Esse apoio refere-se ao matriciamento ou apoio matricial, que é um suporte realizado por profissionais de diversas áreas especializadas, oferecido a uma equipe interdisciplinar com a finalidade de ampliar e qualificar as ações das UBS (BRASIL, 2011b). Os profissionais do matriciamento podem realizar acompanhamento e atendimento no território, sem que precisem acessar outros serviços.

O grupo de matriciamento, se organizou, está indo nas unidades, de acordo com as suas possibilidades. Estão tentando (os profissionais) fazer esse link, estão tentando fazer esses contatos com os outros serviços. (P13).

Conforme o depoimento, acima, o apoio matricial acontece de acordo com as possibilidades dos profissionais de saírem do serviço, pois eles trabalham com uma equipe mínima de profissionais. O matriciamento ocorre quando a equipe de referência da UBS sente a necessidade de apoio da saúde mental para abordar e dirigir um caso que demande, por exemplo, o esclarecimento do diagnóstico, tanto para o usuário quanto para a sua família. Oferece suporte na realização de grupos de usuários de drogas e seus familiares (AZEVEDO, 2012; OLIVEIRA, CAMPOS, 2017).

Com relação ao matriciamento, percebe-se que o acompanhamento ao usuário precisa ser em seu território, pois no seu tratamento deve ser priorizado o convívio com a sua família e comunidade. Também trabalhar na perspectiva da prevenção e da proteção da saúde no seu contexto de vida.

Interações que pudesse trabalhar na comunidade, no território. Olhar aquele usuário como um todo e não como um portador de uma doença. (P1) Então, tem toda a parte da comunidade, esses trabalhos junto à comunidade, a parte de prevenção e promoção de saúde, o regaste da cidadania. (P6).

Os profissionais de saúde da $A B$ necessitam atuar diretamente com a população no seu território. Para tanto, não devem se restringir a estrutura física da $A B$. Isto porque se identifica que as necessidades de saúde revelam-se com o convívio no coletivo, nas visitas domiciliares. Assim, essas necessidades identificadas e acolhidas no território, poderão ser suscetíveis a ações (GONDIM, 2011; BRASIL, 2012b).

Eles estão no território e, é lá que a gente tem que conseguir lidar com eles, trabalhar com eles (usuários de drogas). Só que isso ainda é um sonho em muitos municípios e tal. A gente precisaria estar trabalhando no território, no campinho de futebol da comunidade. (P11).

O trabalho realizado pelos profissionais da atenção básica, por ser ofertado no território possibilita o conhecimento das reais necessidades daquele local e tem potencial para ofertar cuidado que seja resolutivo. Além disto, 
tem-se a possibilidade de realizar um trabalho atento e minucioso direcionando-se ao diálogo e escuta para atender as demandas dos usuários (BRASIL, 2012b). Isto possibilita conhecer a singularidade, o contexto, os modos de viver desses usuários e, assim, amplia a compreensão do profissional sobre quais ações ofertar (FEUERWERKER, 2014).

Quem sabe tu passas numa escuta e conversa (fala sobre um atendimento realizado) com um colega meu, porque de repente tu vieste porque está precisando conversar. (P3).

A escuta auxilia na identificação de um problema ou uma situação que pode não ser aparente, mas no decorrer da conversa o usuário passa a confiar no profissional e traz a sua real inquietação. Para que isso aconteça, o profissional precisa estar disposto a escutar e estar bem consigo.

Para ti chegar sentar com um usuário e fazer uma escuta de qualidade tu precisa estar bem. Isso é fundamental! Ninguém escuta ninguém tendo questões suas para trabalhar. Isso quer dizer, que tem que estar sempre sorrindo? Não, mas não pode estar, por exemplo, adoecido [...] e tu vê o quão importante escutar o usuário a partir da singularidade dele. (P11).

Os profissionais de saúde adoecidos podem ser um obstáculo para a efetivação da realização da assistência inviabilizando uma boa escuta em prol do estabelecimento de um vínculo que se reverta em benefícios a quem é atendido. A escuta faz parte da proposta da $\mathrm{PNH}$, na qual é importante para a formulação de vínculo entre profissional-usuário. Os profissionais de saúde ao realizarem uma escuta qualificada apreendem às demandas e necessidades dos usuários permitindo a efetividade das práticas de saúde (BROTTO, 2012; BRASIL, 2013; SIQUEIRA et al., 2019).

Além da escuta, tem-se o vínculo que aproxima e estabelece uma relação de confiança e responsabilidade entre o usuário e o profissional (VIEGAS, 2012), que é compreendido como um fator positivo para a atenção à saúde, como refere o profissional:

Apostar no vínculo [...] porque a gente sabe que nas nossas relações é mais fácil a gente efetivar alguma coisa quando a gente tem esse vínculo, quando a gente tem essa troca esse feedback, assim, sabe o que está acontecendo de construir o plano junto com ele. (P1).

A partir das discussões, percebe-se que os profissionais de saúde buscam prestar a assistência em consonância com a $\mathrm{PNH}$, pois eles priorizam oferecer suporte especializado aqueles da ABS. Isto tem sido desenvolvido por meio do matriciamento, pois eles identificam a necessidade de atuação nos territórios. Além disto, os profissionais dos CAPS buscam realizar uma escuta com responsabilidade e que seja resolutiva. 


\section{Considerações finais}

Ao apreender as concepções de profissionais de saúde de Centro de Atenção Psicossocial álcool e Drogas evidenciou-se que atenção à saúde dos usuários de álcool e outras drogas existe modos diferentes de prestar atenção: um na perspectiva da abstinência e outro na redução de danos.

No que se referem às políticas públicas de saúde, os profissionais percebem que há um desconhecimento, dificuldade em articular a teoria com a prática e uma assistência ainda pautada na lógica reducionista e fragmentada. Porém, alguns buscam uma prática de trabalho em consonância com a Política Nacional de Humanização. Também, mostram as dificuldades da gestão do município na comunicação com os serviços.

Para isso, é fundamental que os profissionais se organizem e se mobilizem para que os responsáveis pelas políticas públicas de saúde, bem como, os gestores do município, conheçam as fragilidades e potencialidades dos serviços de saúde. Além disto, torna-se importante elucidar as concepções dos profissionais de saúde dos CAPS ad, com vistas a realizar o planejamento e desenvolvimento de estratégias e implementação de mudanças para as ações de saúde mental que sejam voltadas as necessidades dos usuários de drogas.

\section{Referências}

ASSIS, J.T.; BARREIROS, G.B.; CONCEIÇÃO, M.I.G. A internação para usuários de drogas: diálogos com a reforma psiquiátrica. Rev. Latinoam. Psicopat. Fund., v.16, n.4, p. 584-596, 2013.

AZEVEDO, E. B. de.; FILHA, M. de. O. F. Práticas inclusivas na rede de atenção à saúde mental: entre dificuldades e facilidades. Revista Ciência \& Saúde, Porto Alegre, v. 5, n. 2, p. 60-70, 2012.

BRASIL. Ministério da Saúde. Secretaria de Atenção à Saúde. SVS/CN-DST/AIDS. A Política do Ministério da Saúde para Atenção Integral a Usuários de Álcool e outras Drogas/Ministério da Saúde. 2.ed. rev. ampl.Brasília:Ministério da Saúde, 2004.

BRASIL. Gabinete de Segurança Institucional. Conselho Nacional Antidrogas. Política Nacional sobre Drogas. Brasília, 2005.

BRASIL. Ministério da Saúde. Secretaria de Gestão do Trabalho e da Educação na Saúde. Departamento de Gestão da Educação na Saúde. Residência multiprofissional em saúde: experiências, avanços e desafios / Ministério da Saúde, Secretaria de Gestão do Trabalho e da Educação na Saúde, Departamento de Gestão da Educação em Saúde. - Brasília: Ministério da Saúde, 2006.

BRASIL. Ministério da Saúde. Secretaria de Gestão do Trabalho e da Educação na Saúde. Departamento de Gestão da Educação em Saúde. Política Nacional de Educação Permanente em Saúde / Brasília: Ministério da Saúde, 2009.

BRASIL. Ministério da Saúde. Secretaria de Atenção à Saúde. Núcleo Técnico da Política Nacional de Humanização. Acolhimento nas práticas de produção de saúde. (2 ed., 5 reimp.). Brasília, DF: Ministério da Saúde, 2010.

BRASIL. Politica Nacional De Humanização. 1ª edição. 1ª reimpressão. Brasília - DF 2013.

BESSA, M.A. Contribuição à discussão sobre a legalização de drogas. Ciência \& Saúde Coletiva, v. 15, n. 3, p. 632636, 2010. 
ATENÇÃO À SAÚDE: CONCEPÇÕES DE PROFISSIONAIS DO CENTRO DE ATENÇÃO PSICOSSOCIAL ÁLCOOL E DROGAS

BRASIL. Ministério da Saúde. Portaria n 3.088, de 23 de dezembro de 2011. Institui a Rede de Atenção Psicossocial para pessoas com sofrimento ou transtorno mental e com necessidades decorrentes do uso de crack, álcool e outras drogas, no âmbito do Sistema Único, 2011a.

BRASIL. Ministério da Saúde: Guia prático de matriciamento em saúde mental / Dulce Helena Chiaverini (Organizadora). [et al.]. [Brasília, DF]: Ministério da Saúde: Centro de Estudo e Pesquisa em Saúde Coletiva, 236 p. $2011 b$.

BRASIL. Conselho Nacional de Saúde. Diretrizes e normas regulamentadoras de pesquisa em seres humanos. Resolução $\mathbf{n}^{\circ} \mathbf{4 6 6}$, de 12 de dezembro de 2012a.

BRASIL. Ministério da Saúde. Secretaria de Atenção à Saúde. Departamento de Atenção Básica. Acolhimento à demanda espontânea: queixas mais comuns na Atenção Básica / Ministério da Saúde, Secretaria de Atenção à Saúde, Departamento de Atenção Básica. - Brasília : Ministério da Saúde. (Cadernos de Atenção Básica n. 28, Volume II). 2012b.

BROTTO, T. C. de A.; DALBELLO-ARAUJO, M. É inerente ao trabalho em saúde o adoecimento de seu trabalhador? Rev. bras. Saúde ocup, São Paulo, v. 37, n. 126, p. 290-305, 2012.

CECCIM, R.B. Educação permanente em saúde: desafio ambicioso e necessário. Interface Comun Saúde Educ, vol. 9, n 16, p. 161-77, 2005.

CLEMENTE, A.; LAVRADOR, M.C.C.; ROMANHOLI, A.C. Desafios da rede de atenção psicossocial: problematização de uma experiência acerca da implantação de novos dispositivos de álcool e outras drogas na rede de saúde mental da cidade de Vitória-ES. Polis e Psique, Vol . 3, n.1, 2013.

CORTES, L.F.; TERRA, M.G.; PIRES, F.B.; HEINRICH, J.; MACHADO, K.L.; WEILLER, T.H.; et al. Atenção a usuários de álcool e outras drogas e os limites da composição de redes. Rev. Eletr. Enf. V. 16. n. 1 p. 84-92, 2014.

CRUZ, M. S. Redução de Danos, Prevenção e Assistência. Prevenção ao uso indevido de drogas: Capacitação para Conselheiros e Lideranças Comunitárias. - 4. ed. - Brasília : Ministério da Justiça. Secretaria Nacional de Políticas sobre Drogas - SENAD, 2011.

FERNANDES, A. T; CASTRO, C; MARON, J. Desafios para implementação de Políticas Públicas: intersetorialidade e regionalização. Centro de Convenções Ulysses Guimarães Brasília/DF. 2013.

FEUERWERKER, L. C. M. Micropolítica e saúde: produção. 1ª Edição. Porto Alegre, Editora Rede UNIDA, 2014.

FORTESKI, R.; FARIA, J. G. de. Estratégias de Redução de Danos: um exercício de equidade e cidadania na atenção a usuários de drogas. Rev. Saúde Públ., Florianópolis, v. 6, n. 2, p. 78-91, 2013.

GANEV, E.; LIMA, W. de. L. Reinserção Social: processo que implica continuidade e cooperação. Revista Serviço Social \& Saúde. UNICAMP Campinas, v. X, n. 11, 2011.

GIL, A. C. Métodos e técnicas de pesquisa social. $6^{a} e d$. São Paulo: Atlas S.A. 2008.

GONDIM, R. et al. Organização da atenção. In: Gondim R, Grabois V, Mendes Junior WV, organizadores. Qualificação dos Gestores do SUS. 2. ed. Rio de Janeiro, 2011. p. 93-120.

GUEDES, A. C. et al. A mudança nas práticas em saúde mental e a desinstitucionalização: uma revisão integrativa. Rev. Eletr. Enf. [Internet]. V.12, n. 3, p. 547-53, 2010.

LAVRAS, C. Atenção Primária à Saúde e a Organização de Redes Regionais de Atenção à Saúde no Brasil. Saúde Soc. São Paulo, v. 20, n. 4, p. 867-874, 2011.

LORENZETTI, J.; LANZONI, G.M.M.; ASSUITI, L.F.C.; PIRES, D.E.P.; RAMOS, F.R.S. Gestão em saúde no Brasil: diálogo com gestores públicos e privados. Texto Contexto Enferm, Florianópolis; Vol. 23 n.2 p. 417-25, 2014.

MAYER, R. T. da. R.; ALBERTI, A. Z.; SIMONE, A. de. A.; GASPAR, F. L.; MINOZZO, F. Cartilha de Redução de Danos para Agentes Comunitários de Saúde, 2013.

MEDINA, M. G. et al. Promoção da saúde e prevenção de doenças crônicas: o que fazem as equipes de Saúde da Família? Saúde Debate, Rio de Janeiro, v. 38, n. especial, p. 69-82, 2014.

MINAYO, M. C. S. O desafio do conhecimento: pesquisa qualitativa em saúde. 14ª ed. São Paulo: Hucitec, 2014.

NASCIMENTO, S. do. Reflexões sobre a intersetorialidade entre as políticas públicas. Serv. Soc. Soc, São Paulo, n. 101, p. 95-120, 2010. 
OLIVEIRA, M.M.; CAMPOS, G.W.S. Formação para apoio matricial: percepção dos profissionais sobre processos de formação. Rev. Saúde Coletiva, Rio de Janeiro, v. 27, n. 2, p. 187-206, 2017.

PASSOS, E. H; SOUZA, T. P. Redução de danos e saúde pública: construções alternativas à política global de "guerra às drogas". Psicologia \& Sociedade; v. 23, n. 1, p. 154-162, 2011.

PEREIRA, M. O.; VARGAS, D.; OlIVEIRA, M. A. F. de. SMAD, Rev. Eletrônica Saúde Mental Álcool Drog. (Ed. port.). Ribeirão Preto, v. 8, n.1, 2012.

RIBEIRO, L. M. et al. Saúde mental e enfermagem na estratégia saúde da família: como estão atuando os enfermeiros? Rev Esc Enferm USP, v. 44, n. 2, p. 376-82, 2010.

SENAD. Glossário de álcool e drogas / Tradução e notas: J. M. Bertolote. Brasília: Secretaria Nacional de Políticas sobre Drogas, 2010.

SPRICIGO, J, S; ALENCASTRE, M, B. O enfermeiro de unidade básica de saúde e o usuário de drogas - um estudo em Biguaçú-SC. Rev. Latino-Am. Enfermagem, v. 12, n. especial, p. 427-32, 2004.

SILVA, K. L. et al. Intersetorialidade, determinantes socioambientais e promoção da saúde. Ciência \& Saúde Coletiva, v. 19, n.11, p. 4361-4370, 2014.

SIQUEIRA, D.F.; TERRA, M.G.; VIEIRA, L.B.; MORESCHI, C.; MELLO, A.L.; SOCCOL, K.L.S. Ações de cuidado aos familiaresde usuários de substâncias psicoativas: perspectivas de profissionais e familiares. Texto Contexto Enferm, v. 28, 20180022, p. 1-14, 2019.

SODELLI, M. A abordagem proibicionista em desconstrução: compreensão fenomenológica existencial do uso de drogas. Ciência \& Saúde Coletiva, v.15, n. 3, p. 637-644, 2010.

SOUZA, M. C. de. et al. Integralidade na atenção à saúde: um olhar da Equipe de Saúde da Família sobre a fisioterapia. O Mundo da Saúde, São Paulo, v. 36, n. 3, p. 452-460, 2012.

TOLENTINO, D.M.; MELLO, A.L.; SIQUEIRA, D.F.; TERRA, M.G. (Re)conhecimento dos profissionais da saúde sobre a reforma psiquiátrica. Revista Saúde (Sta. Maria)., Vol. 44, n. 2, p. 1-11, 2018.

TORCATO, C. E. M. et al. Drogas e Sociedade. Sau. \& Transf. Soc, Florianópolis, v.4, n.2, p.i-iv, 2013.

VARGAS, et al. Representação social de enfermeiros de centros de atenção psicossocial em álcool e drogas (CAPS AD) sobre o dependente químico. Esc. Anna Nery, Rio de Janeiro, v.17, n.2, 2013

VIEGAS, S. M. F.; PENNA, C. M. M. O vínculo como diretriz para a construção da integralidade na estratégia saúde da família. Rev Rene. v. 13, n. 2, p. 375-85, 2012.

VAZ, F. C. A.; CUNHA, T. C.; OLIVEIRA, D. C. Dificuldades na implementação das ações em saúde do trabalhador pelo Sistema Único de Saúde. Revista Interdisciplinar NOVAFAPI, Teresina, v.4, n.4, p. 61-65, 2011. 